\title{
Les fondements de la Socioéconométrie
}

\author{
Taladidia Thiombiano*
}

\section{Résumé}

Cet article se présente comme un approfondissement de la réflexion dans mon œuvre Vers une approche en économie politique : la Socioéconométrie (1997). C’est une réflexion épistémologique relative à la construction de l'économie fondée sur le fait économique. Nous essayons de montrer qu'au regard de la complexité de ce fait économique qui, à l'origine est un acte social, il est important pour comprendre la réalité économique de se fonder sur la démarche multidimensionnelle. À cet effet, nous avons rappelé quelques constructions de théories économiques et les faits qui ont permis ces constructions. Un examen approfondi de ces théories montre l'importance de l'espace et du temps. Toutefois, il ne s'agit pas du temps et de l'espace classiques, mais des concepts vus sous l'angle de leur dépérissement ou non dans leur attribution à l'économie. La combinaison espace/temps permet de mieux comprendre la réalité économique en tant que totalité systémique. La systématisation du fait économique conduit à considérer tout fait économique comme ayant des considérations sociologiques et tout fait sociologique comme ayant des incidences économiques. Enfin, la réflexion nous a conduit à examiner le rôle de l’Économétrie comme support dans l'approfondissement et la compréhension des théories économiques.

\section{Abstract}

This article aims to deepen the reflection in my work, Vers une approche en économie politique : la Socioéconométrie [Towards a political economic approach : Socioeconometrics] published in 1997, which discusses the construction of the economy based on economic facts. With regard to the complexity of economic facts, which at the beginning is a social act, the article attempts to show that it is important to understand economic realities through a

* Centre d'Etudes, de Documentation, de Recherche Economiques et Sociales (CEDRES) de l’Université de Ouagadougou, Burkina Faso. 
multidimensional approach. An in-depth examination of the construction of some economic theories and facts that helped those constructions to happen shows the importance of the concept of space and time. Yet, it is not about the classical concept of time and space, but about concepts perceived under the angle of their decline or not within the economy. The combination of space and time helps to better understand economic realities as a systemic totality. The systematisation of economic realities leads to a consideration of economic facts as having some sociological implications, and sociological facts as having some economic incidences. Lastly, the reflection then examines the role of econometrics as a means to deepen and understand economic theories.

«L’analyse économique n’a jamais été le produit d’une curiosité intellectuelle détachée, relative au pourquoi des phénomènes sociaux, mais celui d'un besoin pressant de reconstruire un monde qui suscite un profond mécontentement »

Friedrich Von Hayek

\section{Introduction}

La Socioéconomérie voudrait dépasser le cloisonnement des sciences. C’est pourquoi elle fait appel au-delà du fait économique, à l'essence cachée des choses, notamment au fait social. Toutefois, comme le souligne Durkheim (1895), les faits sociaux doivent être regardés comme des faits de la nature. Ce qui met au cœur du débat, la méthode des lois de la nature et celle des lois économiques. Pour qu'il en soit ainsi, il faut une démarche objective reposant sur trois piliers : l'écartement de tout jugement a priori des faits, le regroupement des faits d'après leurs caractères extérieurs communs, et l'appréhension des faits par le côté où ils se présentent, isolés de leurs manifestations individuelles.

L'économie en tant que science, étudie des actes ayant un caractère social. De ce fait, il est difficile de séparer l’économie du support social qui en est la cause. Ce qui ne veut pas dire qu'elle n'a pas une construction objective, lui donnant sa scientificité. Cette construction est basée sur des lois, des postulats, des théorèmes. Ces lois peuvent elles se construire in abstracto en dehors des lois sociales comme semblaient le faire l'Ecole néoclassique pour qui l'Economie s’apparente aux phénomènes naturels ? Dans ce cas, les lois qui en découlent sont des lois de la nature. Certains comme Cournot, Walras, Pareto en viennent à comparer l’Économie à la science la plus pure, à savoir les mathématiques. Ainsi, le livre de Cournot intitulé « Recherches sur les principes mathématiques de la théorie des richesses » de 1838 en dit long.

Cette similitude comme on le voit, s’éloigne du fait réel pour se confiner dans une généralisation scientifique qui tend à enlever à l'Economie son ca- 
ractère de fait social donc de fait non naturel. Cette conception n'est pas tout à fait exacte car quand on lit l'ouvrage de Cournot, on voit que son objectif est d'analyser la richesse des nations à la lumière de l'outil mathématique. Ainsi, dans cet ouvrage, il traite de l'impôt, de la valeur d'échange, de la concurrence des producteurs, des marchés et du revenu social. Somme toute, il essaie de comprendre les actes économiques à travers la mesure. Ainsi, il dit, « Admettons donc que le débit ou la demande annuelle D est, pour chaque denrée, une fonction particulière $F(p)$ du prix de cette denrée. Connaître la forme de cette fonction, ce serait connaître ce que nous appelons la loi de la demande ou du débit ». Il poursuit en disant qu' " elle dépend évidemment du mode d'utilité de la chose, de la nature des services qu'elle peut rendre ou des jouissances qu'elle procure, des habitudes et des mœurs de chaque peuple, de la richesse moyenne et de l'échelle suivant laquelle la richesse est répartie». On voit bien que Cournot qu'on dit être trop abstrait fait dépendre la loi de la demande non seulement de l'utilité du bien mais aussi des habitudes et mœurs de chaque peuple.

Le présent papier ne revient pas sur la définition de la socioéconométrie faite lors du Colloque de Ouagadougou en 1997. Il vise à donner les fondements du paradigme à travers un certain nombre de questionnements ${ }^{1}$. On dira que la Socioéconométrie est une recherche de la compréhension de l'Économique à l'aide de nouveaux instruments développés par la recherche scientifique. C'est une approche systémique du fait économique dans l'espace et dans le temps. C'est une démarche épistémologique qui vise à enrichir l'analyse économique grâce à la prise en compte des faits sociaux qui structurent les réactions des agents aux variations de leur environnement.

Dans les développements qui vont suivre, il sera examiné successivement : le fait économique, la multidisciplinarité de la réalité économique, le temps et l'espace économiques, et enfin la systématisation du fait économique.

On peut alors se poser la question qu'est-ce qu'un fait économique?

\section{Le fait économique}

\section{Définition}

Le fait économique est le résultat d'actes économiques individuels ou collectifs dans le cadre d'un processus de production, de distribution ou de consommation. Comme le soulignait Karl Marx (1859), c'est un processus qui met en relation les hommes entre eux d'une part, et d'autre part, les hommes avec la nature. Ce rapport avec la nature est-il un acte naturel ou un acte social ? Par acte naturel, il s’agit de savoir si cela résulte d'une loi physique ; par contre, par acte social, il s’agit d'un comportement résultant d'une acti- 
vité de l'homme. Si on accepte cette distinction, il est évident qu'il s'agit d'un acte social qui vise à satisfaire un certain nombre de besoins objectifs et/ou subjectifs. Nous sommes dans ce cas loin des lois naturelles qui sont indépendantes de la volonté humaine même si avec les actes économiques l'homme contribue aujourd'hui à la modification de ces lois de la nature. Nous en voulons pour preuve les changements climatiques qui sont intervenus progressivement au cours des siècles suite aux agressions de l'homme contre la nature. D’ailleurs, la théorie du chaos, née dans les années 1970 a révolutionné la science. En effet, elle a démontré que « des systèmes simples engendrent un comportement complexe et que des systèmes complexes engendrent un comportement simple » (James Gleick 1987). Apparemment, les systèmes simples de production ont entraîné des comportements complexes à savoir l'accumulation et le profit. Ce désir effréné a conduit à toujours chercher à améliorer les systèmes de production d'où à exploiter toujours davantage les ressources naturelles. Finalement, il en résulte que l'acte économique ne peut pas être un acte isolé. Il se situe dans le cadre d'un système à la fois social et naturel. De ce fait, pour comprendre au plan scientifique ces différents actes, individuels et collectifs, il faut les inscrire dans un cadre systémique et d'interdépendance des phénomènes. C'est dans ce souci de compréhension que l'on cherche à déboucher sur des lois économiques, c'est-à-dire des explications générales des actes économiques.

\section{L'Économique}

L'économique est l'explication scientifique des actes individuels et/ou collectifs dans un contexte de généralisation dans le cadre des processus de production, de répartition et de consommation. Le marché constitue un des cadres privilégiés de compréhension de ces actes. Toute l'économie moderne ne peut se comprendre au plan théorique que dans le cadre de ce marché. Mais ce marché lui-même n'est pas apparu ex-nihilo. C'est parce qu'est apparu l'échange marchand entre les hommes qu'est née la théorie du marché, c'est-à-dire de l'Offre et de la Demande. Dès lors, l'économique ferait difficilement abstraction de son objet, à savoir comprendre et expliquer les logiques des actes économiques. Si tel est son objet, peut-on assimiler l'économique à la physique ou aux mathématiques et partant, ses lois aux lois naturelles ? Nous venons de parler de lois économiques, et comme toute démarche scientifique, l'analyse économique procède par un essai de connaissance des phénomènes qui engendrent ses actes. Cette recherche de connaissance consiste d'un point de vue méthodologique à dépasser les cas particuliers qui engendrent ces actes pour les placer dans un cadre général, c'est-à-dire dans une totalité. En nous référant à l'étude des lois de la nature, nous savons qu'il existe deux méthodes différentes pour les étudier. Une 
première consiste à partir des faits et des observations que grâce à l'expérience les scientifiques effectuent, et cherchent des lois et des modèles mathématiques pour en rendre compte. Par la suite, les lois sont généralisées pour que le formalisme puisse englober de plus en plus de faits observés. Cette façon peut être qualifiée de phénoménologie. Tel que le fait remarquer le physicien Chérif Zananiri (2002), « il faut bien comprendre que cela ne veut nullement dire que le formalisme mathématique est simple ou facilement saisissable ». Dans le cas de cette méthode, la mécanique quantique part de l'observation et construit un ensemble que l'on peut transposer dans le monde macroscopique. La seconde méthode pour approcher la nature prend le chemin inverse. Cette fois, elle part des grands principes naturels. En fait, on part de postulats et on construit à partir de là, tout un édifice composé de théorèmes. La relativité d'Einstein est l'exemple type de cette méthode a priori. Ainsi, sa relativité cherche à donner aux lois une forme qui perdure indépendamment du mouvement des observateurs.

La généralisation née de ces actes conduit à dégager des lois, des théorèmes et à poser comme condition que toute chose est égale par ailleurs. Ainsi en est-il des lois de fonctionnement du marché : lieu où circulent librement les marchandises ; concurrence pure et parfaite ; non-intervention de l’État, etc. Mais si nous reprenons la formule toute chose étant égale par ailleurs, ce dernier postulat est à la fois vrai et faux. Vrai car au plan scientifique comme en mathématique et dans les lois de la nature, on admet qu'il n’y a pas changement de conditions de l'expérience. Toujours en se référant à Einstein, ce dernier dit qu'il est « très frustrant de considérer que deux observateurs embarqués sur des référentiels différents ne puissent pas formuler les lois de la même façon. S’il en est ainsi, les lois économiques se vérifient et s’appliquent partout où il en est. Faux, car l'immobilité des phénomènes naturels et sociaux relève d'une faible probabilité. Certainement, les lois naturelles changent beaucoup plus lentement que les lois économiques et sociales; mais elles changent malgré tout. La considération des changements est alors comme une question de degré. Lorsqu'il devient quasi certain, le degré d’incertitude de la loi augmente et son universalité diminue car il devient difficile d'avoir des conditions identiques en tout lieu et en tout temps. Malgré cette incertitude, les spécialistes des sciences sociales et principalement de l'Économique, formulent des hypothèses, dégagent des lois pour mieux appréhender la réalité économique. De toutes les façons, même les mathématiques n’échappent pas à cette règle de créer des conditions pour mieux résoudre certaines difficultés de la réalité. Ainsi en est-il de l'introduction des nombres complexes lorsque la réalité a mis en face du mathématicien la recherche de la racine carrée de nombre négatif. Pour contourner la difficulté, il inventa le nombre 
complexe. Ainsi par exemple, dans la pratique, en physique, si on considère un point de l'espace-temps comme un évènement, il est défini par le référentiel $(x, y, z, t)$ avec les conditions suivantes : $x_{1}=x, x_{2}=y, x_{3}=z, x_{4}=i c t$ avec $i^{2}=-1$.

Le fait économique est complexe dans la mesure où il est le plus souvent le résultat d'actes sociaux qui mettent en rapports une série d'acteurs $y$ compris la nature. Les économistes en formulant des lois économiques essaient de comprendre cette complexité dans un cadre multidimensionnel.

\section{La multidimensionnalité de la réalité économique}

\section{L'analyse multidimensionnelle}

Auparavant, nous avons dit qu'il était difficile dans le domaine économique d'avoir en tout lieu et en tout temps des conditions identiques à l'instar des sciences de la nature. C'est cette différence qui a conduit de nombreux auteurs tel que Jean-Louis Guigou (1977) à recourir à l'analyse multidimensionnelle pour mieux saisir les phénomènes économiques. Il fait remarquer que dans « les sciences exactes, le réel s’impose de l'extérieur (exemple : un volcan, la mer, la chute d'un corps...) il n’en est pas de même dans les sciences humaines. En Économie, le réel ne doit pas se confondre avec l'apparent. Le réel dépend de l'imagination des gens. C'est ce que Walras (1874) appelle les sciences psychico-mathématiques du fait qu'elles ne sont pas extérieures à nous. Donc, dans les sciences humaines, la première démarche consiste à définir le réel que l’on veut étudier. À cet égard, la pensée scientifique moderne, l'anthropologie structurale et les outils qui leur sont associés définissent le réel comme une structure, c’est-à-dire « un ensemble de propriétés considérées comme caractéristiques entre les éléments». Cette méthode s’oppose à la linéarité de la pensée humaine. De ce fait, la pensée est unidimensionnelle alors que comme le soulignent F. Wasservogel et J. Lechat (1971), la réalité appartient à un espace de dimension infinie. L'unidimensionnalité de la pensée vient de ce qu’on ne peut pas penser deux choses en même temps. C’est pourquoi J. Fourastié (1975) estime que «l'infirmité majeure du raisonnement rationnel c'est sa linéarité ». Cette critique de la linéarisation a été faite dans les années 1970 par les théoriciens du chaos. Selon James Gleick (1987), sur un graphe, les relations linéaires se représentent par une droite. On voit là effectivement le raisonnement ordonné de l'esprit humain. Ainsi, les relations linéaires ont une signification simple, elles admettent des solutions. Pour Gleick, c'est à cause de cela qu'on en rencontre autant dans les manuels. Les systèmes linéaires dit-il offrent l'important avantage d’être décomposables : on peut les démontrer et les reconstituer, leurs éléments s’emboîtent. 
Les systèmes non linéaires, ne sont généralement pas solubles et ne peuvent pas s'additionner. Il ajoute qu'en dynamique et en mécanique des fluides, on tend généralement à éliminer les termes non linéaires pour obtenir une compréhension claire et facile des phénomènes. Tel qu'on le voit, ce n’est pas seulement l'économiste qui a recours à la linéarisation des relations.

Nous devons reconnaître que la formation de notre esprit est telle que nous déformons de façon fondamentale la réalité aussi bien naturelle que sociale. À cet effet, nous pouvons nous référer à la découverte en mars 1979 de deux quasars qui a intrigué le milieu scientifique, car le couple semblait présenter des spectres lumineux quasi semblables. De nombreuses recherches ont fini par montrer que ces deux jumeaux ne sont que deux images d'un même objet à travers une lentille, en reprenant le modèle optique. Dans un tel cas, selon les physiciens, c'est l'optique gravitationnelle qui a joué des tours aux scientifiques.

\section{Le raisonnement économique classique}

Les systèmes de production, de distribution et de consommation s'intensifient, mettant en œuvre les individus, les ménages, les communautés, les nations voire les continents. La nature elle-même n'échappe pas au renforcement et à cette multiplication des relations. Le Sunami qui a secoué l'Asie du Sud-Est a été ressenti jusqu'aux côtes de l'Afrique de l'Est. En terme d'analyse systémique ou multidimensionnelle, on peut penser que quelqu'un comme F. Quesnay fut un précurseur avec son Tableau Économique pour montrer les liens qui existent entre les différentes parties d'une économie nationale à l'image du corps humain. Quesnay est un médecin et il s'inspire du fonctionnement du corps humain qu'il connaît bien pour le transposer au corps économique. Cela nous amène à nous poser la question, qu'est ce qui constitue la base du raisonnement économique ?

Considérons Adam Smith, sa théorie de la valeur basée sur la valeur travail est une réaction aux thèses mercantilistes et physiocratiques qui font reposer la richesse des nations respectivement sur l'accumulation des métaux précieux et sur l'agriculture. Lorsque les mercantilistes disent que la richesse des nations repose sur l'accumulation des métaux précieux, c'est parce que l'Espagne, le Portugal, l'Angleterre étaient à cette époque des puissances maritimes et leurs navires qui allaient au-delà du continent et singulièrement en Amérique latine leur permettaient de ramener ces métaux précieux. Quant aux physiocrates français, la réalité navale de leur pays ne leur permettait pas de soutenir une telle position, car la France n'était pas une grande puissance maritime. Au plan agricole par contre, elle avait des atouts. D'ailleurs, cette position agricole de la France existe toujours et l'opposition 
entre la Grande-Bretagne et cette dernière à propos des subventions accordées à l'une et à l'autre des deux puissances au sein de l'Union européenne s'explique en partie par ces différences d'atouts. Incontestablement, il apparaît que l'essai de théorisation économique rentre bien dans une réponse à la richesse des nations, donc aux faits sociaux, puisque ces essais répondent aux préoccupations des sociétés de l'époque et dans un cadre national. C'est la primauté de la nation ou la naissance de la macroéconomie. En réaction à la fois à cette macroéconomie et à cette définition de la théorie de la valeur, l'École classique répond à la question de la richesse par la valeur travail ; toutefois, elle a besoin d'un cadre, d'un système, voire d'un référentiel dans lequel sa logique économique doit fonctionner, à savoir : le système de marché libre. Ce paradigme est le fondement idéologique sur lequel va se construire toute la théorie économique classique et néo-classique. C'est pourquoi, lorsqu'on fait de la modélisation économétrique en faisant abstraction du contexte dans lequel le modèle a été construit, on doit s'attendre à une non-signification d'un nombre important de variables contenues dans le modèle. Quelquefois, il en résulte des paradoxes sans qu'on se rende compte qu'il s'agit de paradoxes et non de résultats faux. Dans la plupart des pays africains, les entreprises de production sont souvent en situation de monopole ou d'oligopole. Dès lors, l'hypothèse formulée dès le départ dans le modèle sur la situation de concurrence pure et parfaite ne peut être vérifiée.

La seconde base de construction de l'économie classique, c'est l'individu. Cet individu dit rationnel, qui cherche son intérêt, est le seul capable de mettre en valeur son travail. Ce principe est une réaction contre l'étatisme des mercantilistes et des physiocrates, mais aussi une façon de contribuer à l'émergence d'une nouvelle classe d'entrepreneurs incarnés par la bourgeoisie porteuse d'espoir au XIXe siècle. Adam Smith et l'École classique ne présentent pas apparemment leur démarche dans un cadre idéologique, mais dans une construction scientifique cohérente. L'ouvrage de Smith Recherches sur la nature et les causes de la richesse des nations (1776) passe en revue dans ses cinq livres, toutes les activités sociales allant des causes qui ont perfectionné le travail jusqu'au revenu du souverain en passant par la théorie de l'accumulation des capitaux. Il y consacre même un chapitre au système colonial dans lequel il défend la liberté des nations. Ce traité ne s'adresse pas à une nation bien précise, encore moins à l'Angleterre son pays, elle ne cible pas apparemment une classe spécifique. Elle montre tout simplement que la richesse des nations comme des individus repose sur la valeur travail de chaque citoyen qui, a priori cherche son intérêt personnel ; d'où la portée générale de l'œuvre. Lorsque Smith prône le libéralisme économique, ce n'est pas de façon dogmatique, mais dans le cadre d'une démonstration fondée sur les faits, sans toutefois faire référence à cette réalité 
sociale ; d'où la naissance de la théorie de la valeur travail. Pour que son système fonctionne, il faut un espace où circulent librement les différentes marchandises et ce cadre c'est le marché. Toutes les autres démonstrations telles que celles relatives au capital, au taux d'intérêt, au niveau des prix, vont reposer sur ce cadre.

Existe-t-il une théorie économique en dehors ou indépendamment des faits ? Il est évident que la richesse des nations est une réaction au macrocosme du XIIe au XVIIIe siècles. C'est aussi une réaction à l'encontre de la classe féodale qui maintient les forces productives et notamment l'homme dans l'oppression. L’École classique libère ces forces productives sans déclencher de conflits armés. Au demeurant, Adam Smith condamne le maintien des colonies et aussi de l'esclavagisme, toutes choses contraires à la libération de la force de travail. Dans le chapitre VII du Livre V, il écrit : « les bons traitements rendent l'esclave non seulement plus fidèle, mais plus intelligent, et par conséquent plus utile... ».

Il est aussi bon de rappeler qu'au plan scientifique, au XVIIIe siècle et au début du XIXe siècle, l'analogie dominante est la biologie. Cet aspect ressort chez F. Quesnay, A. Smith ou J. B. Say, pour qui l'économie politique est la « physiologie du corps social ». Ce sentiment est partagé aussi par un groupe de philosophes français qui développent une "physiologie sociale ». Cette vision biologique de l’économie se retrouve également chez K. Marx et chez A. Marshall.

Toujours au sujet des faits, la théorie ricardienne de la rente foncière n'est-elle pas un exemple ? Elle a traversé les temps et reste encore valable dans nos économies avec l'accroissement de la population qui met constamment en valeur des terres marginales. À cet effet, Ricardo dans ses Principes de l'Économie Politique et de l'Impôt (1817), Chapitre II, écrit que « À chaque accroissement de la population qui force un peuple à cultiver des terres d'une qualité inférieure pour en tirer des subsistances, le loyer des terrains supérieurs hausse ». Dans le même temps, bien que la loi de Malthus sur la population ait eu une interprétation plus politique que scientifique, elle n'en demeure pas moins une construction théorique que les faits confirment depuis trois siècles. Les néo-classiques se sont-ils réellement écartés de ces faits pour leur construction théorique puisque leur démarche est hypothético-déductive?

\section{Comprendre les bases de la construction de l'École néo-classique}

On ne peut comprendre l'École néo-classique qu'en rappelant le contexte dans lequel foisonnent les idées intellectuelles à cette époque. De façon générale, comme le souligne Jean Marie Huriot (1994), l'important dans cette seconde moitié du XIXe siècle, c'est l'introduction du raisonnement mathématique dans les sciences sociales. Tout comme A. Smith réagissait contre 
les mercantilistes et les physiocrates à propos de l'origine de la richesse, de même certains auteurs néo-classiques réagissent contre les classiques sur cette même question et plus précisément sur la valeur d'un bien qui selon eux n'est pas fondée sur la quantité de travail mais sur l'utilité de ce bien. Les classiques privilégient l'offre du bien alors que les néo-classiques accordent davantage d'importance à la demande du bien. On ne peut véritablement comprendre tout le raisonnement néo-classique qu’à travers cette primauté de la demande caractérisée par la théorie des préférences individuelles. Elle est au cœur de la rationalité maximisatrice. Ainsi, l’acteur économique, et donc social est construit comme «....un être universel, abstrait et donc asocial, ou encore sans aucune obligation a priori vis-à-vis d'autrui ». C'est une négation de la nature sociale de l’être humain. Pour déterminer une fonction d'utilité, on doit supposer que les préférences vérifient les axiomes suivants :

- les préférences vérifient un pré-ordre complet des choix possibles, ce qui implique la transitivité (si je préfère $a$ à $b$ et $b$ à $c$ alors je préfère $a$ à $c$ ) et la réflexivité. L’agent peut de ce fait hiérarchiser les différents éléments entre lesquels il doit choisir ;

- les préférences sont continues (soit $a>b>c$, il se dégage une combinaison de possibilités $a$ et $c$ qui est indifférent par rapport à $b$ ) ;

- les caractéristiques de ces préférences se fondent sur l'axiome de non-saturation (si la consommation d'une quantité $a_{1}$ de $A$ engendre une utilité $u_{1}$, alors si $a_{2>} a_{1}$ l'utilité de $u_{2}>u_{1}$ ) ;

- l'indépendance des préférences qui est caractérisée par la considération suivante : étant donné les possibilités $a, b$ et $c$ telles que $a>b$, une combinaison de $a$ et $c$ sera préférée à la même combinaison de $b$ et $c$;

- monotonie des préférences dans le temps car l'utilité pour un individu est continue sur l'ensemble de la période et n'est pas affectée par la proximité de retournement de tendances. La réflexion de base est atemporelle ;

- la représentation de courbes d'indifférence résultant des mélanges de possibilités avec comme caractéristiques, la convexité de la fonction générale.

Il se dégage de cette construction, un raisonnement totalement mathématique. En fait, ces développements des néo-classiques constituent la base de l'individualisme méthodologique en opposition à l'holisme méthodologique. 
Le dernier point qui permet de comprendre toute la démarche scientifique de cette école, c'est le bouillonnement scientifique de l'époque à la fois au plan philosophique et au plan des sciences exactes. C'est en réaction au microcosme des néo-classiques devant l'impossibilité pour ce raisonnement de résoudre les problèmes posés par le fait économique (crise de 1929) de l'époque qu'un certain Keynes développe sa théorie générale.

\section{Les fondements de la Théorie générale}

Étant préoccupé par le rôle des faits dans la pensée économique, tout naturellement, nous nous posons la question de savoir si La théorie générale de l'emploi, de l'intérêt et de la monnaie (1936) aurait vu le jour s'il n'y avait pas eu la crise de 1929. Tous les auteurs (Jean de Largentaye) qui ont lu Keynes ou qui l'ont traduit sont d'accord pour dire que son œuvre est une théorie de l'emploi. Mais pourquoi, une théorie de l'emploi ? Parce que la Grande crise de 1929 a mis au chômage des millions de travailleurs. En avril 1930, il y a aux États-Unis, 2429000 hommes et femmes sans emploi et 758000 personnes mises en congés forcés, sans salaire, pour une durée indéterminée. En Allemagne, entre 1930 et 1932, le nombre de chômeurs passe de 3 millions à 6 millions. En Angleterre, pays de Keynes, le nombre de sans- emploi, atteint 2714000 en 1931. Les faits parlent d'eux-mêmes. Mais pourquoi, Keynes, face à une situation réelle, cherche des réflexions théoriques au lieu de recommandations de politiques économiques? En effet, Keynes se préoccupe de trouver les fondements théoriques propres à assurer le « plein emploi ». D’ailleurs, dès le départ, il est clair sur son orientation. Il dit dans la première édition anglaise de sa préface :

Ce livre s'adresse surtout à nos confrères économistes. Nous souhaitons qu'il puisse être intelligible à d'autres personnes. Mais il a pour objet principal l'étude de questions théoriques difficiles et il ne traite qu'à titre subsidiaire l'application de la théorie aux faits.

Tel que l'on pouvait s'y attendre, Keynes commence son ouvrage par une analyse critique de l'économie classique (Livre I). Il écrit que :

Quant au fameux optimisme de la théorie économique traditionnelle, optimisme en raison duquel on a fini par considérer les économistes comme des Candide, qui, ayant abandonné le monde pour cultiver leur jardin, enseignent que tout est pour le mieux dans le meilleur des mondes possibles pourvu qu'on le laisse aller tout seul, il a pour origine, selon nous, la méconnaissance de l'obstacle qui peut être opposé à la prospérité par l'insuffisance de la 'demande effective'.

À travers cette remarque, Keynes dénonce la main invisible des classiques d'une part, et par sa demande effective, c'est-à-dire les dépenses qui peu- 
vent être effectivement réalisées par les agents économiques (sauf l’État) à un moment donné, il veut relancer d'autre part, la consommation pour stimuler la production afin de résorber le problème de l'emploi. C'est la raison pour laquelle, il interrompt la suite logique de son ouvrage pour consacrer le Livre II à des « définitions et concepts » et en particulier à la prévision en tant que déterminant du volume de la production et de la consommation. Pour lui, « les deux vices marquants du monde économique où nous vivons sont le premier que le plein emploi n’y est pas assuré, le second que la répartition de la fortune et du revenu y est arbitraire et manque d'équité ». A ces deux interrogations, il répond à la seconde question en disant que « dans les conditions contemporaines, la croissance de la richesse, loin de dépendre de l'abstinence des milieux aisés, comme on le croit en général, a plus de chance d'être contrariée par elle ». Pour quelle raison ? Parce que tant qu'il n'y a pas plein emploi, une faible " propension à consommer » et donc une forte épargne, n'entraîne pas une augmentation de l'investissement. D'un point de vue purement économique, mieux vaut distribuer de meilleurs salaires aux individus les plus pauvres qui les consommeront.

Pour rompre définitivement avec «la main invisible », il consacre un Chapitre au rôle de l'État à qui il demande d'agir sur la consommation et l'investissement qui constituent les deux volets de la " demande effective " grâce à sa politique fiscale, exercée par le canal du taux d'intérêt. Cette politique permet à l'État d'exercer une influence positive sur « la propension à consommer ».

En conclusion, parti d'un fait économique qui est la crise économique, Keynes élabore une théorie générale dont l'objectif est de contribuer à résoudre cette crise, et certainement d'autres dans le futur. Mais, il ne s'agit pas d'une crise conjoncturelle, mais d'une crise structurelle qui a attaqué les fondements mêmes du système libéral. La Théorie générale, n'est pas une politique économique, car elle ne s'adresse ni à un pays, ni à l'Europe, elle recherche les origines de la crise et les moyens scientifiques de la conjurer. Une des nouveautés de Keynes est l'introduction de la prévision qui se rapporte au temps.

\section{Le temps et l'espace économiques}

Le temps et l'espace constituent deux éléments fondamentaux dans la formation et l'explication des théories économiques. Mais avant d'en venir à des cas spécifiques, les scientifiques se sont toujours posés des questions : Qu'est-ce que l'espace ? Qu'est-ce que le temps ? Sont-ils liés ? 


\section{L'espace}

L’espace économique est un champ dans lequel se déroulent les différentes activités économiques. Avec la naissance du marché, cet espace est devenu très ouvert. Il s'étend désormais au-delà du cadre national. Même l'analyse d'une économie villageoise exige aujourd'hui pour sa compréhension et son fonctionnement qu'elle se fasse dans un cadre ouvert. Il s'agit d'espaces géographiques, qui ne nous intéressent que partiellement dans ce cadre. Ce qui nous intéresse dans le contexte actuel ce sont les espaces liés à la théorie économique. Nous retiendrons deux types d'espace : l'espace fini et l'espace infini.

\section{L'espace infini}

L’espace infini est celui utilisé par la théorie. En effet, la théorie a une portée générale, elle ne peut délimiter des frontières pour montrer là où les relations, les actions se déroulent. Il n’y a pas d'attribut en tant que tel. La théorie se caractérise par son impersonnalisation. Ainsi, quand A. Smith intitule son œuvre La recherche de la véritable cause de la richesse des nations, il ne précise pas quelle nation et dans quel continent ? La théorie donne ici l'impression de quelque chose d'incolore et d'inodore. De même, lorsqu'il parle de la valeur travail, il ne délimite pas à une catégorie sociale bien déterminée. C'est cette forme de démarche que nous qualifions d'espace infini. Nous en avons fait la démonstration plus haut dans la présentation du contexte de la naissance de la Théorie générale de Keynes.

\section{L'espace fini}

À l'inverse, l'espace fini se définit dans le cadre de l'application de la théorie. C'est désormais un champs précis auquel on à faire face afin d'appliquer la théorie. C'est un espace clos, même si on doit tenir compte des échanges dans lesquels se déroulent les événements. Cet espace est un espace social dont chaque cadre a ses spécificités propres. C’est pourquoi même l'action individuelle ne peut se comprendre en dehors de cet espace dans lequel elle est et qui l'influence ; et sur lequel, en retour, il peut avoir également une certaine influence. Vu ainsi, la querelle individualisme méthodologique et holisme méthodologique ne se justifie pas dans un contexte d'interaction. En d'autres termes, c'est la compréhension de la microéconomie à travers la macroéconomie. Mais, l'inverse est aussi indispensable, c'est-à-dire, l'incidence des actes individuels sur les actes collectifs, d’où la compréhension de la macroéconomie à travers la microéconomie.

L'espace infini de la théorie économique fait passer de l'invisible, de l'abstrait à l'espace fini, c'est-à-dire au concret, au visible. Mais peut-on parler d’espace sans se référer au temps? 


\section{Le temps}

Le temps économique à l'instar de l'espace économique peut se comprendre à deux niveaux.

\section{Le temps infini}

La caractéristique d'une théorie est qu'elle doit traverser le temps. C'est ce qui donne à cette théorie sa portée générale et atemporelle. Le temps infini se rapporte à la théorie économique, en ce sens que cette dernière n'est pas définie par rapport à une période donnée. Le temps économique infini se caractérise par le mouvement. Il se distingue du temps historique. Selon Fernand Dumont (1970), les économistes procèdent à plusieurs types de découpages qui sont entre autres :

- périodes objectives qui se réfèrent aux données qui s’imposent aux agents. Ici, il peut s’agir de délais techniques de production ;

- et périodes subjectives qui renvoient aux plans de ces agents, c'est-àdire à des délais de réaction de la part par exemple du consommateur.

Il peut s'agir aussi comme le fait G. Granger (1955) de la distinction entre temps réversible et temps irréversible.

En fait, il ne s'agit pas de ces types de temps. Le temps infini est un temps ayant une portée générale. Tel que le souligne Léna Soler (2000), toutes les activités humaines ont une histoire, mais la science a ceci de particulier qu'elle prétend à la vérité. Or poursuit-il, « la vérité est, par essence même, atemporelle. Si donc la science est bien un ensemble de vérités, elle ne saurait être soumise au temps ». En réalité, ce temps qui n'existe pas dans la science n'est qu'apparent. Le temps dans la science est borné à gauche, car toute théorie a un début. L'infini est relatif au côté droit, c'est-à-dire à l'avenir. Le temps fait appel aux faits.

Nous pouvons nous demander si la Théorie de la valeur travail qui est le fondement de la richesse des nations a dépéri après plus de deux siècles ? Mais il est évident que la période où $\mathrm{A}$. Smith fait sa découverte, n'est plus la même que celle d'aujourd'hui et comme le dit Héraclite « on ne se baigne jamais deux fois dans la même rivière ». Tout de même, les sociétés en développement se reposent la même question "Quelle est la nature et les causes de la richesse des nations ? » La réponse reste toujours la même, « la valeur travail ». Tout en n'ayant pas le même temps économique deux fois, il n'en demeure pas moins vrai qu'on peut avoir les mêmes causes $n$ fois et comme l'adage le dit «les mêmes causes produisent les mêmes effets ». 


\section{Le temps fini}

Ce que nous qualifions de temps fini relève du domaine empirique des sciences. C'est le temps empirique et à l'opposé du temps théorique qui est borné à gauche, le temps empirique est fermé. Considérons l'intitulé suivant, faisant l'objet d'une recherche « L'économie du Burkina Faso ». Un tel énoncé est incomplet car on ne sait pas sur quelle période porte l'étude. Il est important de dire à quelle période dans l'existence de cette nation ; à quel moment de son histoire veut on appréhender son économie ?

Dans cet exemple, on se pose la question de savoir si la définition de l'espace ne contraint pas à définir le temps. À l'inverse, la définition du temps économique n’entraîne-t-elle pas la définition de l'espace sous-jacent. Ce n’est pas toujours évident. En effet, lorsque l'économiste français François Perroux intitule son ouvrage L'Économie du XX e siècle, il délimite le temps qu'il borne à droite et à gauche sans cependant délimiter l'espace. Est-ce que cet espace n'est pas sous-entendu car il s'agit de l'Économie des nations existantes au XXe siècle ou ce que l'on appelle aujourd'hui l'Économie mondiale. Le temps fini ne délimite t-il pas par projection l'espace ?

En attendant des recherches plus fouillées, nous nous interrogeons sur le rôle du temps dans le comportement de certaines sociétés. Au regard de leurs attitudes, on est conduit à se demander si ces communautés ne considèrent pas le temps comme infini. S'il en est ainsi, cette attitude n'expliquet-elle pas en partie leur sous-développement ? Comment comprendre qu'un pays comme Haïti, indépendant depuis plus de 200 ans, soit un des pays les plus pauvres de la planète ?

\section{Liaison espace et temps économiques}

Souvenons-nous qu'il y a encore quelque temps, l'espace était représenté par trois dimensions (3D) sans le temps. L'introduction du temps par les physiciens donnait une représentation à $4 \mathrm{D}$, ce qui compliquait la représentation. Aussi, la quatrième dimension spatiale a-elle été comprimée de telle sorte que les trois dimensions spatiales n'en font plus que deux, et la troisième est réservée au temps qui est représenté par un plan en mouvement. L’Économie n’a-t-elle pas grâce aussi aux mathématiques, réalisé cette liaison ? En effet, la théorie des données de panel marque à notre sens ce trait d'union espace-temps. Mais notre propos en Économie n'est pas à ce niveau. Notre interrogation sur la question espace/temps est de voir si la théorie économique combine espace et temps infinis pour lui conférer tout son caractère universel.

Dans la réalité, dans une discipline sociale comme l’économie, peut-il réellement exister un espace infini dans la mesure où la théorie n’est pas indépendante du fait économique tel que nous l'avons explicitée auparavant. 
Si tel est le cas, elle n'est pas indépendante de l’objet économique. De plus, le sujet lui-même, c’est-à-dire le concepteur de la théorie est lui-même partie intégrante de la réalité analysée. En considérant le subconscient du sujet, nous sommes en définitive dans une situation d'espace infini fini. Considérons Keynes dans sa Théorie générale au Chapitre sur l’État. Il écrit ceci « Aucune argumentation convaincante n’a été développée qui justifierait un socialisme d’État, embrassant la majeure partie de la vie économique de la communauté. Ce n’est pas la propriété des moyens de production dont il importe que l'État se charge ». Il est évident que cette position le place hors du contexte scientifique qui a prévalu jusqu’à présent. De même, la théorie abstraite de la valeur s'appuyait, à l’origine, sur des bases idéologiques. Elle représentait en un certain sens, la rationalisation d'une société économique où régnait presque sans entraves le capitalisme concurrentiel. Mais ce support n’entraînait pas, de soi, la disqualification de l'axiomatisation : celle-ci n’était pas une simple concrétisation des attitudes des capitalistes de l'époque. En rejetant la complexité des réalités sociologiques, le marginalisme n'avait d'autre recours que de tout expliquer par rapport au subjectif. Il s'agit comme le souligne Fernand Dumont (1970), d'une subjectivité logique. Somme toute, pour lui, la rationalité de la théorie marginaliste se mire dans la rationalité du sujet économique.

La démarche de l'infini est néanmoins indispensable pour construire une théorie objective et universelle. Cette universalité de la théorie qui lui confère son caractère spatial ne saurait être statique. Elle doit, après avoir franchi les frontières, traverser le temps pour consolider son caractère de loi. Néanmoins, on s'interroge sur l'existence d'un temps infini. Il est évident que si les faits sont en rapport avec les sociétés qui sont en perpétuelle mutation, le temps économique ne peut dans ce cas être infini pour une théorie puisque la validité de celle-ci est fonction de l’hypothèse formulée au départ. Que vaut la loi de l'offre et de la demande si l'hypothèse de « concurrence pure et parfaite n’est pas vérifiée » ? Le temps théorique infini, finit avec le dépérissement du cadre qui lui a donné naissance, dans le cas présent, le système libéral. La démarche hypothético-déductive utilisée par les économistes est empruntée aux «sciences dures », notamment les mathématiques. C’est surtout dans ce domaine que l'hypothèse est plus forte. Ainsi, dans le système décimal, $1+2=3$. Dans les « sciences géomanciques », la relation mathématique est $1+2=1$. En effet, dans ce système qui est binaire, le $2=$ 0 . Dès lors, toute science a besoin comme on le voit pour sa systématisation de construire un cadre, de formuler des hypothèses, ainsi en est-il de l'économique. 
Les développements scientifiques récents ont permis, au plan économétrique, de combiner l'espace et le temps pour mieux comprendre la réalité économique. Cette méthode repose sur les données de panel. Nous y voyons déjà les prémisses de la socioéconométrie.

Le fait économique conduit à parler de l'espace mais aussi du temps. Tels sont les trois concepts fondamentaux qui définissent en partie les contours de la socioéconométrie.

\section{La systématisation du fait économique}

Le fait n’a de sens dit Maurice Niveau (1970) que « dans la mesure où il est repéré et analysé en vue d’éclairer les ressorts de l'activité économique. L’économiste poursuit-t-il, doit s'appuyer sur l'histoire, non seulement pour y découvrir le passé mais aussi pour mieux comprendre le présent et anticiper l'avenir ». Le fait économique en tant que résultat d'un acte social ne peut se comprendre que par rapport à l'espace et par rapport au temps. Il va permettre de montrer les relations symbiotiques et symboliques qu'il y a entre les hommes d'une part, et entre les hommes et la nature, d'autre part. Au-delà de ces relations historiques et séculaires, se sont développées d'autres relations engendrées par les systèmes de production, de répartition et de consommation. Ces nouvelles relations sont celles qui sont nées entre nations, entre continents dans le cadre de ce qu'on appelle aujourd'hui la mondialisation.

Pour comprendre le fait économique, il faut se situer dans le cadre de ces relations de causalité. Il faut essayer de relier les phénomènes entre eux de façon à comprendre « qui cause qui » et si au besoin il n’y a pas une causalité réciproque ou un effet de feedback entre ces phénomènes. Le fait économique fait référence au temps, à l'histoire, pour reprendre l'expression de Maurice Niveau. En effet, il est important de savoir quelle est l'influence du passé sur le présent et ce quelle pourrait être dans l'avenir. On se préoccupe de connaître pour la même période de quelle manière les actions, les prises de décision individuelles et collectives, institutionnelles sont susceptibles d'agir sur les comportements de certaines variables économiques. Comme en physique, regardons les phénomènes d’induction électromagnétique. Une variation locale (dans l'espace de $E$ ) produit une variation temporelle de $B$ qui produit un autre terme de champ électrique, qui à son tour apporte une contribution supplémentaire au champ $B$, et ainsi de suite. En Économie, on observe les mêmes réactions à la fois spatiale et temporelle. Comment par exemple l'évolution à la hausse des cours du pétrole va-t-elle agir sur la croissance de l'économie mondiale, qui à son tour va agir sur la richesse des nations ? Depuis quelques années, un débat existe parmi les économètres sur 
l'idée avancée par Granger (1969) et reprise par Sims (1980) selon laquelle dans un modèle économétrique, toutes les variables explicatives doivent être considérées comme exogènes. Ils rejettent la distinction entre variables exogènes et variables endogènes dans la partie explicative. Pour ces auteurs, la détermination de la causalité doit permettre d'orienter le sens de l'influence d'une variable sur une autre. Du reste, ces problèmes de relations causales avaient déjà été posés au plan mathématique par Cramer. En physique, le principe des inégalités de Heisenberg s'inscrit en quelque sorte en faux contre l'existence de relations causales. Il souligne que les événements ont lieu dans le monde sans cause : si nous savons pourquoi une substance est radioactive, il ne nous est pas possible de prédire le moment exact de l'émission, et lorsque celle-ci se produit, elle semble avoir lieu sans aucune raison.

De façon générale, il convient, pour comprendre le fait économique, de faire une sociologie de ce fait et inversement de procéder à une économie du fait sociologique.

\section{Faire une sociologie du fait économique}

Faire une sociologie du fait économique consiste à comprendre l'acte économique dans un espace social. Il faut replacer l'acte économique dans le cadre systémique qu'est la société. Considérons le fait économique suivant : vente par les paysans sahéliens de leurs produits agricoles à des prix bas au moment des récoltes tout en sachant que quelques mois plus tard dans l'année, ces produits seront beaucoup plus chers et qu'ils vont les racheter ainsi. Au sens marginaliste du terme, il y a irrationalité économique de la part de ces paysans dans le contexte des lois du marché. Pour comprendre ces paysans dans leur comportement, il faut se situer dans le contexte de la théorie du revenu-objectif que nous avons développée, il y a déjà quelques années (T. Thiombiano 1996). Dans le même ordre d’idée il faut se référer à la courbe atypique d' « offre du travailleur à objectif » de E. Berg (1962).

Le principe du revenu-objectif conduit le paysan ou tout autre agent économique placé dans ce contexte précis à offrir davantage ses produits quand les prix baissent de façon à atteindre le revenu désiré pour la satisfaction de ses besoins ponctuels. A l'inverse, si les prix augmentent, il peut diminuer les quantités offertes, une fois le revenu-objectif atteint. Il s'agit là d'un paradoxe, en contradiction avec la théorie libérale de l'offre et de la demande dans une situation de concurrence pure et parfaite.

La sociologie du fait économique amène l'économiste à reconnaître le caractère social de tout acte économique qu'il soit individuel ou collectif. Cette démarche entraîne la nécessité de connaître le milieu, l'espace dans lequel l'acte économique se réalise ou a été réalisé. Au-delà de l'acte économique, on cherche à connaître l'essence cachée des choses. Cette quête de 
connaissance de l'essence cachée conduit à analyser le fait économique sous un angle sociologique. Étant donné le problème de causalité dont nous avons parlé, il est important de procéder à une économie du fait sociologique.

\section{Procéder à une économie du fait social}

Le fait social peut être chargé d'une dimension économique. C’est pourquoi, il est indispensable à travers le fait sociologique de chercher l'aspect économique qui est susceptible d'en découler. La guerre 1939-1945 est un fait sociologique dont les véritables motifs pourraient se comprendre à travers la crise économique et financière de 1929. Historiquement, la coopération entre les hommes apparaît comme un fait social. Dans la réalité, à travers cette coopération il a toujours existé des rapports de production, de distribution et de consommation. Aujourd'hui, les courants migratoires de jeunes Africains prêts à braver le désert, les fils barbelés de l'Occident ne peuvent se comprendre qu'à la lumière des problèmes économiques que connaît le continent africain. Le chômage est le motif principal. Ce n'est pas le goût de l'aventure maritime qui les attire, encore moins le froid du Canada ou de l'Alaska, mais davantage, échapper à la misère et à la pauvreté. De ce fait, tout comme les richesses de l'Afrique ont conduit à sa colonisation, de même les richesses de l'Europe attirent aujourd'hui la jeunesse africaine comme les fleurs attirent les abeilles. L'arrêt de ce flux migratoire ne peut être résolu que par la résolution des questions économiques qui sous-tendent ces migrations. L'intégrisme religieux qui se développe n’est pas un fait simplement sociologique du début du XXIe siècle, il ne peut s’interpréter qu'à travers l'appauvrissement d'une jeunesse de plus en plus en proie au chômage et à la misère. Et comme le disait Marx, c'est parmi les pauvres que la religion recrute ses adeptes. Plus prêt de nous, les récents événements des banlieues françaises, tout en étant un fait social, ne peuvent s’arrêter à cette simple interprétation. Les jeunes, pour la plupart issus de familles d'immigrés sont souvent des désœuvrés qui ont quitté l'école très tôt ; de ce fait, ils sont sans emploi. Que ces jeunes trouvent des emplois, aient une formation continue, on verra qu'ils n'auront aucune raison de faire des actes de vandalisme ou de s'adonner à la drogue. Ce type de comportement a déjà été souligné par Raymond Boudon (1984) quand il dégage dans sa relation, phénomènes économiques et phénomènes sociaux, presque la loi suivante : « si les conditions économiques se détériorent $(\mathrm{A})$, alors la violence collective (révoltes, agitation, grèves...) augmente (B) ». C'est là, toute l'importance de faire l'économie du fait sociologique.

L’interaction sociologie et économie remonte le temps. De Marx à Weber, la naissance du capitalisme a poussé les sociologues et les économistes à se pencher sur ce lien. Ainsi, pour le sociologue italien Carlo Trigilia (2002), 
le champ de la sociologie économique peut se définir comme étant caractérisé par « un ensemble d'études et de recherche visant à approfondir les rapports d'interdépendance entre phénomènes économiques et sociaux ». Cela nous ramène au débat sur l'opportunité ou non de l'abstraction, c'està-dire d'une "Économie pure » au sens de Walras. En effet, ce dernier dit que « la théorie pure est la lumière de la théorie appliquée »². V. Pareto (1909), tout en étant un disciple de Walras, ne partage pas entièrement ce radicalisme de Walras. En effet, il ne considère pas les mathématiques comme l'unique méthode scientifique. Pour lui, « la logique courante suffit d'ordinaire pour les relations de cause à effet, tandis qu'il faut souvent employer pour les relations de mutuelle dépendance une forme spéciale de raisonnement logique : à savoir les raisonnements mathématiques ». Il faut rappeler que Pareto a aussi une formation de sociologue.

\section{L'économétrie et la méthodologie de la science économique}

Bien que les interrogations ne datent pas d'aujourd'hui, il convient dans la mesure où le débat reste entier de s'interroger le cas échéant sur le rôle des mathématiques dans la théorie économique. Quelle place doivent-elles prendre dans la démarche scientifique de l'économiste ?

Ces questions se placent aujourd'hui comme le souligne Jean-Marie Huriot (1994) parmi les questions classiques de méthodologie économique. Rappelons que la méthodologie est l'étude de la manière dont les chercheurs élaborent et valident une connaissance scientifique selon la définition de Walliser et Prou (1988).

Ce que le scientifique cherche à faire, c'est expliquer l'existence et le déroulement des phénomènes en vue d'éclairer au besoin l'action. C'est le cas de l'économiste qui réfléchit sur une science d'action. Or expliquer disent A. d'Autume et J. Cartelier (1995), «C'est d'abord établir la validité d'une relation causale, ensuite vérifier que cause et conséquence se trouvent bien dans le cas d'espèce, à ce que la relation causale implique. Mais on ne peut établir objectivement la validité d'une relation causale que si celle-ci a une certaine prétention à l'universalité ». Voilà qui nous éclaire sur la méthodologie scientifique.

Un fait économique n'est pas isolé et c'est ce que nous avons essayé de montrer tout au long de cette étude. Un fait économique est le résultat d'un ensemble d'autres faits qui peuvent être à la fois économique, social, macroscopique, psychologique (individuel), microscopique. Et l'économiste de chercher à expliquer ces faits dans un cadre systémique. C'est pourquoi, Hal R.Varian (1992) dit que la Science économique élabore des modèles des phénomènes sociaux. Il ajoute que par « Modèle, nous entendons une représentation simplifiée de la réalité ». Cela signifie que cette élaboration ne peut 
pas se faire en dehors de cette réalité dont le constructeur lui-même est un sujet. C'est là, une différence de position fondamentale avec le constructeur des lois de la nature. S'il en est ainsi, il faut comprendre qu'il n'y a pas de modèle neutre. Il est le résultat d'hypothèses. Dès lors, la transposition systématique de ces modèles dont la plupart sont construits dans le cadre d'économies de marché peut quelquefois poser des problèmes méthodologiques et partant d'interprétation des résultats de la recherche empirique ou phénoménologique dans le contexte d’économies en développement notamment des économies africaines. Dans ces pays, il y a souvent deux problèmes : la qualité des données et l'absence de concurrence pure et parfaite. L'enthousiasme dans lequel tombent les jeunes chercheurs quant à la certitude de leurs résultats nous amène quelquefois à les tempérer. Mais, de là à déconseiller le recours aux mathématiques pour valider ou pour mieux comprendre une théorie économique ou la réalité économique, c’est un pas que nous n'osons pas franchir. Au contraire, nous les y encourageons mais avec comme recommandation, la prudence et le travail descriptif préalable qu'il faut entreprendre avant de déterminer le modèle économétrique approprié dans le cadre pratique.

L'avantage du recours à l'économétrie, c'est qu'elle se fonde davantage sur l'explication des phénomènes que leur interprétation qui comporte une grande part de subjectivité. En effet, dit Raymond Boudon (1991), dans les sciences humaines, une partie de la recherche relève non de l'explication des phénomènes mais de l'interprétation du vécu. L’économétrie, nous avons dit, est un ensemble de théories et techniques mathématiques, de procédés statistiques dont l’objet est de tester les hypothèses sur les phénomènes économiques, d'estimer les coefficients des relations économiques, de prédire les valeurs probables que prendront les variables dans les processus économiques (T. Thiombiano 2002). Pour D. Henry (1995)³, « l'économétrie est cette approche scientifique visant à la compréhension des aspects économiques de la conduite humaine. Il apparaît effectivement dans cette définition, les aspects économiques de la conduite humaine. Ceci nous situe bien au cœur de la problématique de la socioéconométrie qui voudrait éviter l’interprétation de ces actes économiques de la conduite sociale, pour se situer dans une optique d'explication de ces actes. Mais en même temps, la socioéconométrie montre qu'il est probable que dans chaque fait social, il y ait une explication économique. Au niveau méthodologique, la socioéconométrie place au centre de ses préoccupations, l'importance du temps économique et de l'espace économique en distinguant ce qui est fini et ce qui est infini. Cette méthode permet de faire la distinction entre démarche déductive (Ecole néo-classique en économie ou Einstein sur la relativité) et démarche inductive (l'Ecole classique en économie ou la mécanique quanti- 
que en physique). Selon la méthodologie de l'infirmation de Popper, les théories sont scientifiques, si et seulement si leurs prédictions sont au moins en principe, infirmables.

\section{Conclusion}

Au cœur d'un débat qui a toujours alimenté à chaque époque la science économique, la socioéconométrie voudrait apporter une contribution à la compréhension d'une part, de la construction des théories économiques, d'autre part, montrer l'importance de la multidisciplinarité d'une science carrefour en pleine expansion grâce au développement prodigieux des autres sciences telles que les mathématiques, la physique etc. L'économique est avant tout un acte social qui ne peut se dissocier de la société en général. Aucune théorie économique ne se construit indépendamment des faits, c'est ce que la socioéconométrie tente d'expliquer à travers la mise en exergue du fait économique. Mais cette relation avec les faits ne signifie nullement qu'il n’y a pas une construction scientifique fondée sur la formulation de théories pour expliquer justement ces faits. Derrière chaque fait économique se cache un fait social et derrière chaque fait social, peut se cacher un fait économique. Ainsi, pourrait s’expliquer une opération sociale banale comme le don de femmes dans les sociétés traditionnelles.

Le second aspect que la socioéconométrie voudrait montrer, c'est l'importance de l'usage des mathématiques pour expliquer l'évolution des phénomènes économiques. Cet usage vise à éviter des interprétations qui biaisent la compréhension des faits économiques. L’usage des mathématiques est une condition nécessaire mais non suffisante de scientificité de l'économie. En effet, dans une version modérée, les mathématiques rendent plus aisés à la fois le contrôle de la cohérence interne du discours et la confrontation avec les faits.

L'Économétrie est une branche, voire un instrument de la science économique qui joue un double rôle (T. Thiombiano 2002) :

- instrument de validation de la théorie économique ;

- instrument de prospection et de prévision.

Le troisième aspect de la socioéconométrie qui découle des précédentes analyses, est le recours au temps et à l'espace comme instrument de compréhension du fait économique et son extension pour formuler des théories économiques d'où l'importance de la distinction entre "espace et temps infinis » et « espace et temps finis ». Étant donné l'usage populaire de ces deux concepts que constituent le temps et l'espace, il y a lieu d'entreprendre une recherche sur ces notions de temps économique et d'espace économi- 
que. La physique avec la théorie de la relativité a unifié l'espace et le temps à quatre dimensions et l'a restreint à un espace-temps à trois dimensions. L'économie a unifié l'espace et le temps grâce une fois encore, aux mathématiques par l'intermédiaire de la naissance de l'analyse des données de panel.

Enfin, le dernier point du débat porte sur l'analyse systémique qui doit caractériser la démarche socioéconométrique. Cette problématique est confirmée par la Théorie du chaos qui montre que « tout dans l'univers est lié, et le battement d'une aile de papillon à Pékin peut engendrer des remous dans l'atmosphère de New York ». La grippe aviaire, partie d'un fait naturel d'Asie, a pénétré l’Europe malgré le cordon sanitaire installé par ces pays et aujourd'hui, elle a gagné l'Afrique lointaine de cette Asie.

Il convient en un mot dans toute démarche économique de systématiser le fait économique.

\section{Notes}

1. Suite à ce Colloque international de Ouagadougou, un document a été publié : Thiombiano, T., 1997, Vers une approche en économie politique : la Socioéconométrie, Ouagadougou : Éditions PUO/CASE, Université de Ouagadougou.

2. Walras, 1898, Études d’Économie appliquée, dans les textes choisis de Oulès que rapporte Jean-Marie Huriot 1994.

3. Henry, D, 1995, « Le rôle de l'économétrie dans l'économie scientifique », in Autume, A. et Cartelier, J., L'économie devient-elle une science dure ? Paris : Éditions Economica, Série Grands Débats.

\section{Bibliographie}

Araujo, C. et alii, 2004, Économétrie, Paris : Éditions Bréal. Collection AmphiÉconomie.

Berg, E. J., 1962, “Backward sloping labour supply functions in Dual economies: The Africa case”, Quarterly Journal of Economics.

Blaug, M.,1982, La méthodologie économique, Paris : Éditions Economica.

Boudon, R., 1984, La place du désordre. Critique des théories du changement social, Paris : PUF.

Cournot, A., 1974, Recherches sur les Principes mathématiques de la Théorie des richesses. Paris : Éditions Calmann-Levy. Collection Perspectives de l'Économique.

Autume A. et Cartelier, J.,1995, L'économie devient-elle une science dure ? Paris : Éditions Economica, Série Grands Débats.

Dumont, F., 1970, La dialectique de l'objet économique, Paris : Éditions Anthropos. Durkheim, E., 1895, Les règles de la méthode sociologique, Paris : PUF (1995). Fourastié, J., 1975, Comment mon cerveau s'informe, Paris : Éditions Robert Laffont. 
Gleick, J., 1987, La théorie du chaos. Vers une nouvelle science, Paris : Albin Michel. Collection Nouveaux Horizons.

Granger, C.W. J., 1969, "Investigating causal relations by econometrics models and cross spectral methods”, Econometrica, Vol 37.

Granger, G. G., 1955, Méthodologie économique, Paris : PUF.

Guigou, J.-L., 1977, Méthodes multidimensionnelles. Paris : Dunod.

Henry, D., 1995, « Le rôle de l'économétrie dans l'économie scientifique », in Autume, A. et Cartelier, J., L'économie devient-elle une science dure ? Paris : Éditions Economica, Série Grands Débats.

Huriot, J.-M., 1994, Économie, Mathématiques et Méthodologie, Paris : Economica. Keynes, J. M., 1969), Théorie générale de l'emploi, de l'intérêt et de la monnaie, Paris : Payot.

Largentaye, J., 1969, Petite Bibliothèque, Paris : Payot.

Malthus, R., 1798, Essai sur le principe de population, Paris : Flammarion (1992).

Marshall, A., 1890, Principes d'économie politique, Paris : Editions Gordon et Bench.

Marx, K.,1859, Contribution à la critique de l'économie politique, Paris : Éditions Sociales (1957).

Marx, K., 1867, Le Capital, Paris : Éditions Sociales (1971).

Niveau, M., 1966, Histoire des faits économiques contemporains, Paris : PUF, Collection Thémis.

Pareto, V., 1906, Économie Mathématique, Paris : Édition du Centre d’Économétrie de la Faculté de Droit et des Sciences Économiques de Paris, Tome II. Collection Classique de l'Économie mathématique.

Pareto, V., 1909, Manuel d'Économie politique, Paris : Fayard 2001

Perroux , F., 1960, Économie du XX e siècle, Paris : PUF.

Ricardo, D., 1970, Principes de l'Économie Politique et de l'impôt, Paris : CalmannLevy.

Samuelson, P., 1965, L'économique, Paris : Armand Colin, Collection « U », Tome 1 et 2 .

Sapir, J., 2004, Quelle économie pour le XXI e siècle, Paris : Éditions Odile Jacob, série Économie.

Say, J. B. 1826, Traité d'économie politique, Paris : Calmann-Lévy (5édition 1972).

Sims, C. A., 1980, "Macroeconomics and reality", Revue Econometrica, Vol. 48.

Smith, A., 1976, Recherches sur la nature et les causes de la richesse des nations, Paris : Gallimard, Collection Idées.

Soler, L., 2000, Introduction à l'épistémologie, Paris : Éditions Ellipses.

Thiombiano, T., 1997, «La controverse empirique et théorique posée par le comportement des producteurs-consommateurs », Revue Tiers Monde, $\mathrm{n}^{\circ} 152$, 6, octobre-décembre. 
Thiombiano, T., 1997, Vers une approche en économie politique: la Socioéconométrie, Ouagadougou : Éditions PUO/CASE, Université Ouagadougou, CEDRES.

Thiombiano, T., 2002, Économétrie des modèles dynamiques, Paris : L’Harmattan. Trigila, C., 2002, Sociologie économique. Paris : Armand Colin.

Varian, Hal R., 1992, Introduction à l'analyse microéconomique, Paris : Éditions Boeck Université. Collection Nouveaux Horizons.

Wallisser, B et Prou, C., 1988, La science économique, Paris : Seuil

Walras, L., 1874, Éléments d'Économie politique pure, ou théorie de la richesse sociale, Paris : Editions R. Pichon et R. Durand-Auzias (1952).

Wasservogel, F. et Lechat, J., 1971, «Une méthode de classification d'items qualitatifs ", Riro, $\mathrm{n}^{\circ} \mathrm{V}-2$.

Weber, M., 1922, Économie et société, Paris : Pocket (1995).

Zananiri, C., 2002, La physique pour tous : espace et temps, Paris : Éditions Ellipses.

\section{Compléments de lecture dans le domaine de la Sociologie :}

Boltansky, Luc et Chapello, Eve 1999, Le nouvel esprit du capitalisme, Paris : Gallimard.

Boltansky, Luc et Thevenot, Laurent, 1991, De la justification. Les économies de la grandeur, Paris : Gallimard.

Thevenot, Laurent 2006, L'action au pluriel. Sociologie des régimes d'engagement, Paris, Éditions La Découverte.

Vatin, François 2005, Cinq Essais sur la genèse de la pensée sociologique, Paris, Éditions La Découverte.

\section{Complément de lecture dans le domaine de la Physique :}

Ballian et alii, Roger, 2001, Le temps et ses représentations, Paris : Éditions l'Harmattan.

Hawking, Stephen 2005, Commencement du temps et fin de la physique ? Paris : Éditions Flammarion.

Jonsson, Bodil, 1999, 10 considérations sur le temps, Paris : Editions Gallimard. Lachieze-Rey, Marc, 2003, Au-delà de l'espace et du temps, La nouvelle physique, Paris : Éditions Le Pommier.

\section{Autres disciplines}

Torin, Marc, 2000, Le temps : de la philosophie aux systèmes, Paris : Éditions Flammarion.

Wever, Patrick De et alii, 2002, Le temps mesuré par les sciences, l'homme à l'échelle géologique, Paris : Éditions Vuibert/Muséum National d'Histoire Naturelle.

Zarifian, Philippe 2001, Temps et Modernité. Le temps comme enjeu du monde moderne, Paris : Éditions Flammarion. 